\section{Glow-in-the-dark silk}

If fluorescent green silk becomes the next fashion trend, Hajime Mori and his colleagues at the Kyoto Institute of Technology have it made. In their efforts to develop an insect system to produce foreign proteins, they have engineered a baculovirus that fused the gene coding for one of the chains of the silkworm fibroin gene, the major protein in silk, to the gene encoding green fluorescent protein. When silkworms were infected with the virus, the endogenous fibroin gene was knocked out and replaced with the genetically engineered gene through a process of homologous recombination. As the researchers report in Genes \& Development $(13,511-515$, 1999), the silk glands (see inset) and fiber from the worms containing the fusion gene glowed green. A major advantage of this approach is that the foreign DNA is inserted specifically at the site of the silkworm's own fibroin gene, keeping it under the control of endogenous promoters. Other approaches which use transposons, such as P-elements, integrate foreign genes into random

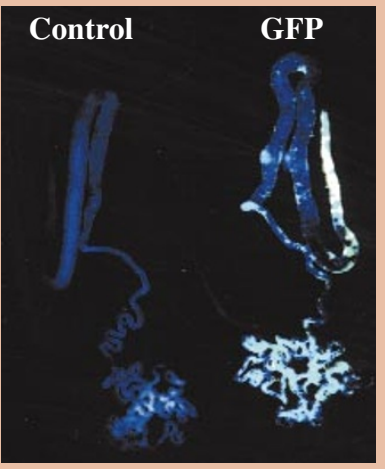
sites in the genome. The fibroin promoter in particular is very strong. "Silkworms can produce $400 \mathrm{mg}$ of silk protein per animal and may be used as an insect factory for the production of many useful proteins," says Mori. For example, replacing the silkworm fibroin gene with the spider gene spidroin could produce spider-like silk, which has many possible uses, ranging from the fibers in bullet-proof vests to parachutes.

\section{Breaking down glycolysis}

By analyzing the behavior of an inducible gene product found in transformed cells, scientists at the Picower Institute for Medical Research (Manhasset, NY) have developed a mechanistic explanation for a phenomenon called the Warburg effect, at the same time identifying a new potential target for anti-cancer therapies. The Warburg effect, discovered over 70 years ago, is the tendency of cancer cells to maintain a high rate of glycolysis, even in the presence of oxygen. The researchers discovered that a previously unknown form of 6phosphofructo-2-kinase (PFK-2), an enzyme with a regulatory role in glycolysis, is expressed at high levels in proliferating tumor cells and is apparently required both for the observed increase in glycolysis and for tumor cell growth. Inhibiting the expression of this inducible form of PFK-2, called iPFK-2, slows the proliferation of the cells in vitro and in vivo in a mouse model. Richard Bucala, senior author on the new study (PNAS 96, 3047-3052, 1999), says that "data thus far [suggests] cancer cells are quite dependent on iPFK-2 for growth." A Seattle based company, Cytokine Networks, plans to pursue commercial development of treatments targeting iPFK-2.

Research News Briefs written by Alka Agrawal, Alan Dove, Alexander Castellino, and Andrew Marshall.

\section{Home-grown arteries}

A collaboration of researchers has succeeded in growing functional arteries from cells derived from a biopsy of cow vascular tissue. By growing the arteries in a novel bioreactor system, they have engineered arteries that are much more resistant to rupture by blood pressure/shear than arteries produced by other methods. To create the vessels, a constant stream of culture medium was pulsed over tube-shaped polymer scaffolds infiltrated with smooth muscle cells. After eight weeks of culture, the vessels were seeded with endothelial cells to mimic the morphology of native vessels. According to Laura Niklason of author on the paper (Science, 284, 489-493, 1999), "To the naked eye, the vessels looked exactly like native vessels." Immunohistological studies confirmed arterial markers were present on the vessels, which contracted properly in response to pharmacological agents. To validate that the arteries were functional, they were grafted into the femoral artery of miniature pigs and their performance compared with grafts prepared using a static system. "The nonpulsed vessels began to show signs of thrombosis by the third week," Niklason observes. "[But] the pulsed vessels remained open for [all] four weeks [of the study]." The approach holds promise as an alternative source of vessels for bypasses in vascular disease. Duke University (Durham, NC), senior

\section{Solid gel sequencing?}

Gel technology appears to be in for a dramatic change. A tiny silicon device honeycombed with microscopic channels could one day replace conventional polyacrylamide gels, according to a talk at the recent meeting of the American Physical Society in Atlanta. Harold Craighead and graduate student, Stephen Turner, from Cornell University (New York) have used electron-beam lithography and materials processing tools to manufacture a silicon chip containing a miniature labyrinth of vertical pillars $(100 \mathrm{~nm}$ thick and $100 \mathrm{~nm}$ apart), analogous to the molecular structure of a gel. By mounting the chip on a slide, the researchers used an optical microscope to monitor the movement (at certain electric fields) of four different fluorescent-labeled DNAs. Preliminary data suggest that shorter molecules are readily separated. The researchers are currently testing different lithographic geometries, including one that has openings of varying diameters ("speed bumps") that will sort DNA fragments by size. One such chip has enabled the Cornell team to separate phage T2 and T7 DNAs within 1000 seconds. According to Craighead, the technique can also be extended to macromolecular protein separation.

\section{Oven-ready nanospheres}

A new technique for fabricating precisely engineered nanometer-scale spheres from silica, developed by researchers at Sandia National Laboratories (Albuquerque, NM) may have a broad range of applications extending from electronics to biotechnology. By mixing the silica with a surfactant and spraying the mixture through a furnace in a fine mist, the scientists were able to form nanospheres ranging in size from $2 \mathrm{~nm}$ to 50 nm. The approach also allows experimenters to vary the size of the pores on the surface of a sphere and to produce intricate structures such as nested spheres. "Individual spherical shells could also be designed to dissolve at a particular physiological pH," explains Jeff Brinker, senior author on the paper (Nature 398, 223-226, 1999). In addition to providing precise delivery vehicles for drugs, the spheres might be used in biosensors or as cages to confine an enzymatic reaction. 